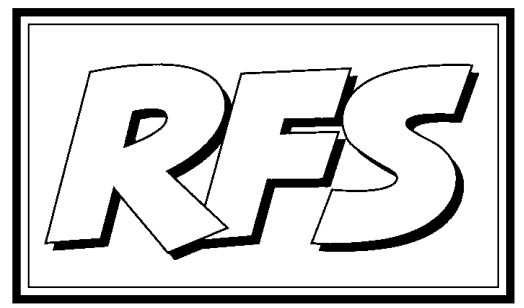

Revista de Fomento Social, 54 (1999), 393-402

\title{
La empresa española en el desarrollo de Iberoamérica: logros y matizaciones
}

La cooperación al desarrollo puede encauzarse por múltiples vías (la ayuda oficial, las organizaciones no gubernamentales, ...). El autor se plantea las posibilidades de cooperación mediante empresas -públicas o privadas-de los países industriales que inviertan en países en desarrollo. Los logros alcanzados y los peligros -cierta incompatibilidad con los criterios de rentabilidad-de esta forma de cooperación se analizan a través del caso de la inversión directa española en Iberoamérica.

Javier GOROSQUIETA REYES, S. J. (*)

(*) Profesor de ICADE. Universidad Pontificia Comillas (Madrid). 
Hay en España una importante empresa, de nombre COFIDES, sigla que significa «Compañía Española de Financiación del Desarrollo, S.A.». Se define a sí misma como «una sociedad anónima de capital mixto cuyo objeto es el fomento de las inversiones productivas de empresas españolas en países menos desarrollados para contribuir, con criterios de rentabilidad, tanto al desarrollo económico de dichos países como a la internacionalización de las propias empresas».

Pero ¿se puede contribuir al desarrollo de un pais pobre o emergente «con criterios de rentabilidad», es decir, dentro de la lógica del mercado? Normalmente estamos acostumbrados a oir hablar de la «ayuda oficial al desarrollo», de carácter concesional, o de los proyectos altruistas de desarrollo de las Organizaciones No Gubernamentales (ONG). Siendo realistas y dada la experiencia, por este doble camino nunca se llegará al desarrollo de los países económicamente atrasados. La ayuda oficial al desarrollo está en estos momentos, a escala mundial, en el 0,32\% del Producto Mundial (en España es el 0,26\% de su Producto Interior Bruto), muy lejos de aquel $0,7 \%$ comprometido en Naciones Unidas allá por el año 1970. Y el movimiento global de dinero del conjunto de las ONGs es de unos 3.500 millones de dólares anuales, muy distante, por ejemplo, de los 88.000 millones de dólares anuales que mueve en créditos sin interés y con un componente de donación del 85\% la Asociación Internacional de Fomento(AIF) del Grupo del Banco Mundial. También esta importante via de las instituciones internacionales para el desarrollo se ha mostrado gravemente insuficiente y limitada. De todas maneras es preciso reconocer la muy significativa labor, en concreto, de las ONG en la financiación de muy numerosos proyectos específicos de desarrollo en países pobres y, tal vez sobre todo, sus empeños y sus logros en la concienciación de la opinión pública en los países ricos en torno a la problemática de la pobreza y del subdesarrollo a escala internacional.

¿Podrá lograrse, finalmente, ese desarrollo por medio de las empresas, públicas y privadas, de los países industriales que inviertan en países emergentes o en desarrollo con criterios de rentabilidad? Creemos que sí, y así lo parece demostrar la experiencia iberoamericana de desarrollo en esta década de los noventa (1).

En este artículo nos limitaremos únicamente a la experiencia inversora de las empresas españolas en Iberoamérica.

(1) Sobre los reales y posibles abusos, por ejemplo, de las empresas multinacionales se matiza al final de este mismo escrito. 


\section{Estrategia y prospectiva}

La presencia de la empresa española en Iberoamérica, y en especial en el Mercosur (Argentina, Brasil, Paraguay, Uruguay), en Chile, en Perú y en Méjico ha aumentado fuertemente estos últimos años, con especial intensidad en los sectores estratégicos de las comunicaciones y del transporte, de la banca y los servicios financieros, de la energía y el turismo. En algunos países como Chile, la empresa española es ahora la primera en cuanto a inversiones europeas, desplazando de este rango a las empresas alemanas, francesas e italianas.

En el pasado, la presencia empresarial española en Iberoamérica, que siempre ha sido importante, se identificaba con la emigración empresarial. Emigraban empresarios que por múltiples razones esperaban encontrar mayores rentabilidades en los nuevos territorios de América que en la vieja España. Ahora, lo realmente nuevo en la relación con Iberoamérica reside en la transferencia de personas morales, empresas, y capitales financieros y tecnológicos apoyados por el desplazamiento temporal de mandos y ejecutivos. España ha descubierto en Iberoamérica los principios básicos de la Inversión Extranjera Directa.

Los fundamentos macroeconómicos de este proceso son obvios: con una peseta fuerte y un excedente de la balanza por cuenta corriente (mercancías más servicios más transferencias), España ha pasado de ser un pais importador de capitales, a tener una capacidad positiva de financiación, y esta capacidad de oferta de ahorro encuentra en Iberoamérica una demanda de inversiones especialmente rentable y adaptada a los conocimientos tecnológicos y organizativos españoles.

Los fundamentos microeconómicos de este proceso también son obvios: la mayoría de los países de Iberoamérica ha optado en estos últimos años por un abandono de las políticas intervencionistas y proteccionistas del pasado, de las políticas también de sustitución de importaciones, y ha promovido privatizaciones y desregulaciones que crean abundantes oportunidades de negocio para las empresas privadas que, al mismo tiempo, van creando el tejido industrial y de desarrollo y van elevando progresivamente el nivel general de vida en los países receptores.

En el momento en el que en Europa el proceso de integración ha dado un salto cualitativo con la introducción del Euro, y en el que Iberoamérica emprende diversos procesos de integración (Mercosur, ALCA -área de libre comercio de las Américas, promovida porEstados Unidos-), es oportuno analizar el futuro de estas 
relaciones entre España e Iberoamérica desde el punto de vista estratégico y prospectivo.

El elemento estratégico más determinante de estos finales del siglo XX es la «globalización», o sea, la tendencia de los mercados y de las tecnologías a elevarse por encima de las fronteras nacionales. La globalización ya actúa plenamente en el sector financiero, con un sistema de mercados interconectados en los que intervienen operadores de todos los centros financieros del mundo; numerosos otros sectores de las materias primas y de las manufacturas también se están constituyendo en forma de mercados globales, con operadores cada dia más «supranacionales».

Las empresas de todos los sectores productivos evolucionan esencialmente en dos direcciones: aumentando su tamaño y formando conglomerados de dimensión mundial, capaces de actuar en mercados globales (fusiones y concentraciones en el mundo de las finanzas, de las telecomunicaciones, del transporte aéreo, del automóvil, etc.), o creando «redes» mediante alianzas que permiten que empresas de menor tamaño encuentren apoyo para su desarrollo global.

La empresa española, encerrada sobre su mercado interior hasta la adhesión a la Comunidad Europea (CE), ha abordado con entusiasmo su «europeización» durante los últimos años, y empieza a contemplar estratégicamente el reto de la globalización. Es en este contexto en el que se establece la importancia de su relación con Iberoamérica.

En la economía de los países en vias de desarrollo, la reciente crisis asiática, de la que todavía no hemos salido del todo, ha puesto en evidencia la transformación pacífica de la economía iberoamericana, la cual, después de una «década perdida» (los ochenta), ha sabido aprovechar el rigor-tal vez excesivo-del ajusteestructural promovido por el Fondo Monetario Internacional (FMI) para poner en marcha un proceso de crecimiento en el que la empresa privada juega un papel de motor, y en el que las administraciones públicas se esfuerzan en facilitar el éxito de la empresa privada. El actual modelo de crecimiento iberoamericano, que acepta la apertura comercial y desmantela las múltiples formas de proteccionismo, apuesta por la competitividad, con la evidente consecuencia macroeconómica de un enorme desequilibrio (momentáneo) entre el ahorro interno y la inversión necesaria; Iberoamérica necesita inversiones extranjeras, y su modelo atrae a los inversores del resto del mundo, ya sea en cartera (compra de acciones) o en proyectos de inversión directa. 
Para la empresa española, la percepción de esta nueva realidad iberoamericana (lejos de las hiperinflaciones tradicionales y de las inestabilidades políticas) es cada dia más nítida, lo que ha permitido identificar algunas grandes oportunidades estratégicas.

La primera se refiere a la imagen tradicional de «España, puente entre Iberoamérica y el resto de Europa», visión tan frecuentemente utilizada en el discurso político desde hace varias décadas, que se transformaría en tópico, pero que ahora comienza a tener aspectos concretos: la «europeización» del sistema productivo español hace que, naturalmente, estas empresas «europeas» utilicen su base española como punto de arranque de sus actividades productivas o comerciales con Iberoamérica. Queda mucho terreno por delante para explotar esta visión estratégica: empresas españolas pueden abrir mercados europeos para los nuevos productores iberoamericanos que hoy son competitivos a nivel internacional; u otras empresas españolas pueden crear, potenciar, promover, redes europeas de empresas interesadas en aprovechar oportunidades en Iberoamérica.

La segunda se refiere a una imagen más contemporánea de «Iberoamérica primer eslabón de la globalización de la empresa española», visión en la que se inscriben, sin duda, las actuaciones que han recibido mayor atención por parte de los medios de comunicación, en estos últimos años (telecomunicaciones, transporte aéreo, electricidad, bancos, etc.). Esta visión estratégica sigue ofreciendo numerosas oportunidades para las grandes empresas españolas, que están empezando a jugar un papel fundamental en el nuevo modelo de crecimiento iberoamericano. (Quien crece es, obviamente, Iberoamérica, a la vez que la inversión exterior obtiene su rentabilidad. Todos contentos). Los éxitos financieros de la mayor parte de esta inversiones (la excepción son las inversiones de Iberia) está demostrando que las oportunidades son reales, altamente rentables, y que permiten unos posicionamientos estratégicos en los que interviene positivamente el factor cultural español. Como ya se pudo verificar en Europa con las inversiones de Estados Unidos en la post-guerra, o en Asia con las inversiones japonesas, cuando existen procesos de integración regional, el inversor extranjero puede desarrollar estrategias regionales antes y mejor que los operadores locales para los cuales estas estrategias se ven frenadas por las tradiciones nacionales. Es así como las empresas españolas están entrando en el sistema productivo iberoamericano, lo están haciendo ya con una estrategia supranacional, apoyándose con frecuencia 
en el Mercosur y en la prospectiva de una gran zona de libre comercio(desaparecen las fronteras comerciales entre las naciones integrantes, pero no se crea una frontera comercial exterior común) para dentro de seis años (ALCA en el 2005).

La tercera visión estratégica ya se refiere a una imagen más estratégica y de futuro «Iberoamérica como elemento de negociación en procesos de globalización», visión en la que se conceptualiza la posibilidad de llegar a acuerdos y alinazas con otras empresas globales del resto del mundo (estadounidenses, canadienses, australianas, japonesas, etc.) en base a la solidez y a las ventajas comparativas de la presencia de la empresa española en Iberoamérica.

Eléxito de las inversiones directas españolas en Iberoamérica, las intervenciones positivas de las empresas españolas en procesos de privatizaciones o en grandes licitaciones, están creando la imagen positiva de una aventura empresarial española en Iberoamérica que «abre puertas» y que tiene credibilidad. En este contexto es fácil contemplar posibles alianzas que proporcionen a empresas del resto del mundo parcelas de poder en Iberoamérica, a cambio de obtener otros campos de actuación para la empresa española.

Las tres visiones estratégicas señaladas apuntan a un desarrollo espectacular de la inversión directa española en Iberoamérica durante los próximos años, y esta evolución, sin duda deseable por sus notables repercusiones sobre la economía española y sobre el desarrollo de Iberoamérica, requiere actuaciones conscientes y pertinentes de las administraciones públicas españolas y de las propias empresas (2).

\section{Expansión}

La dinámica inversora en la región ha permitido que España se haya convertido en el primer inversor europeo en la zona, durante el periodo 1990-1994 y segundo durante el año 1995. En el año 1996 totalizó 1,23 billones de pesetas, un 30\% más que en 1995, pasando a liderar nuevamente la inversión durante 1997, que alcanzó los 1,95 billones de pesetas, lo que representa un aumento del $59 \%$ respecto al año anterior. Es más, lejos de apagarse esta trayectoria, según los datos referidos al

(2) En este apartado hemos seguido el desarrollo del estudio de E. FonTELA (1999): La presencia empresarial española en Iberoamérica. Vectores estratégicos, Centro de Promoción de Inversiones ICEX-CEOE, monográfico sobre Iberoamérica, pp. 87-91. 
primer trimestre de 1998, la inversión española ha despuntado nuevamente, al concentrar 200.000 millones en este continente, representando un $41 \%$ de la inversión total en el exterior que se situó en ese trimestre en 488.000 milllones de pesetas. En el primer semestre de 1999 España encabezó las inversiones en Iberoamérica, período en que la fusión y compra de empresas en la región alcanzó los 33.390 millones de dólares (5,34 billones de pesetas), concentradas básicamente en operaciones en Argentina y Chile.

Según un estudio de la Cámara de Comercio de Santiago de Chile, las inversiones de capitales españoles en la región fueron de 20.579 millones de dólares $(3,29$ billones de pesetas), equivalentes al $62 \%$ del total invertido en Iberoamérica en ese primer semestre.

La fuerte penetración española consiguió arrebatar el liderazgo en materia de inversiones a Estados Unidos, que esta vez sólo llegó al 12\%, con 4.156 millones de dólares (664.960 millones de pesetas). En tercer lugar quedó Méjico, con 1.503 millones de dólares, 240.480 milones de pesetas (4,5\% del total), seguido de Chile (4\%) y Argentina $(3,1 \%)$.

Las inversiones españolas se concentraron fundamentalmente en los sectores energético y bancario-financiero. «Las OPAS (ofertas públicas de acciones) de Endesa España por Enersis y Endesa Chile y de Repsol por YPF en Argentina explican por sí solas la mitad del valor de las operaciones totales de la región», afirma el estudio.

La inversión global directa exterior española ha ido creciendo desde 66.857 millones de pesetas en 1986 a 676.904 en 1994 y a 1.957 .388 millones en 1997. 1998 trajo un nuevo incremento y la tendencia continúa en 1999. Y no se crea que se trata sólo de las grandes empresas como Repsol, Telefónica, Iberdrola, Endesa, Gas Natural, BBV, Santander o BCH, que protagonizan lo más brillante de la inversión. Porque los protagonistas de este boom son las medianas empresas (también pequeñas) de alimentación, auxiliares del automóvil, textiles, de transporte o de servicios (3).

La década de los noventa está ofreciendo, pues, oportunidades diametralmente opuestas a las acaecidas durante los años ochenta. Así se constata que el nuevo

(3) Los datos han sido tomados del trabajo de R. CASILDA (1999): La empresa española en Iberoamérica ante las nuevas exigencias. Una nueva visión estratégica, en la obra citada en la nota (2), pp. 103-113, y del diario El País, 15-7-99. 
modelo económico motiva la entrada de empresas y capitales extranjeros en estos países, al despejarse los graves riesgos que se produjeron años atrás, y que ocasionaron la retirada de numerosas entidades, como es el ejemplo de la banca estadounidense (claramente afectada por la deuda externa en los años ochenta). En estas nuevas oportunidades han convergido varios factores tanto endógenos como exógenos, entre los que destacan el crecimiento espectacular de los mercados internacionales de capitales; la desregulación y las innovaciones financieras; las bajas tasas de interés a corto plazo en Estados Unidos y otros países industriales; la renegociación de la deuda externa de los países iberoamericanos; las reformas económicas emprendidas por éstos, que incluyen la liberalización y privatización de sectores con gran relevancia estratégica para el capital extranjero, como es el caso del finaniero; y la necesidad, por parte del capital y las empresas de los países industriales, entre ellos España, de aproximarse a mercados expansivos como son los iberoamericanos, con muchas necesidades que cubrir de la población, y de desplazarse de mercados maduros, con muchas necesidades satisfechas, como es evidentemente también el caso del mercado español.

España tiene ahí, pues, grandes oportunidades, que en parte son ya realidad, de conseguir buenas rentabilidades mediante la colaboración, intrínseca al proceso, al desarrollo de todas esas economías emergentes o necesitadas de un definitivo despegue.

\section{Matizaciones}

Primera. Si bien esa afluencia de capitales externos ha entrañado indudables beneficios, como el de atemperar la severa restricción de fondos externos que sufrió la región en los años ochenta, también ha tenido efectos indeseados para la estabilidad económica misma y el desarrollo financiero de los países receptores, al orientarse muchos de esos fondos a inversiones a corto plazo que pueden adquirir un carácter especulativo y volátil. Ahí está, por ejemplo, para demostrarlo, la estampida o huida de muchos capitales exteriores desde Brasil, cuando este país fue «contagiado» en 1998 por la crisis financiera y económica internacional que tuvo su origen en el Sureste asiático. Esto incrementó notablemente las dificultades de ese pais para dominar y superar su propia crisis. Es de alabar, en este sentido, el comportamiento de las grandes empresas españolas inversoras en la región, que aguantaron allí la crisis, a pesar de los reveses momentáneos sufridos por sus 
acciones en los mercados de valores.

La existencia de ese capital exterior especulativo y volátil ha despertado un gran interés regional por conocer la calidad, la naturaleza, los efectos y el comportamiento de dichas corrientes financieras. Cabe destacar la labor constante de la Comisión Económica Para América Latina (CEPAL), cuyos especialistas han realizado una amplia gama de investigaciones y documentos al respecto.

Segunda. Las compras de empresas iberoamericas por el capital exterior y las fusiones empresariales pueden llegar a crear situaciones de fuerte oligopolio o de monopolio, contrarios a la competencia y, por lotanto, contrarios al interés general de los ciudadanos de esos países. Es necesario funcionen en los mismos algunos mecanismos o instituciones que vigilen y garanticen, como sucede en los países industriales y en la Unión Europea, la vigencia de una debida competencia económica en los mercados.

Tercera. Hemos hablado, pues, de cómo el sistema de libertad de empresa, buscando la rentabilidad, puede cooperar eficazmente al desarrollo de esa región iberoamericana. No hemos hablado todavía de igualdad relativa interna de los niveles de vida. Es preciso que los gobiernos de esos países, mediante una adecuada política social, y también los sindicatos, con su fuerza negociadora, propugnen con eficacia, progresivamente, una relativa igualdad de los niveles de ingresos. Es esto necesario, que se difundan socialmente los frutos del desarrollo. En este sentido es preferible que esos países se coloquen como meta el capitalismo propio de la Unión Europea, con su Estado del Bienestar, la difusión social de la riqueza creada y la relativa igualdad en los niveles de vida de sus ciudadanos. No el capitalismo de los Estados Unidos, con sus grandes desigualdades sociales, sus contratos basura, su incremento notable de la pobreza, su raquítico Estado del Bienestar.

Cuarta. Las empresas extranjeras que compren empresas o que abran de otra manera, filiales en Iberoamérica, se convierten, por ello mismo, en multinacionales. Y todos conocemos cuáles suelen ser los abusos de tales transnacionales: capacidad de eludir el control y la fiscalidad de los estados locales y someter totalmente los intereses de los países en que se asientan al suyo económicoprivado. Es éste un problema no resuelto. El riesgo está ahí. Es de esperar que progresivamente, a través de instancias internacionales para el gobierno y el juego limpio en la economía internacional, se pueda ir acorralando esta materia. 


\section{Conclusión}

Sin dudaque uno de los principales retos éticos del sigloXXI seráel del desarrollo del llamado Sur del planeta. Hemos hablado del momento esperanzador que atraviesa Iberoamérica, región más que en vías de desarrollo, emergente. Queda la olvidada África y otras muchas regiones pobres del mundo, que todavía están lejos del despegue. No nos centremos tanto en una región que prescindamos de las demás. También tienen derecho a una ayuda y colaboración efectiva.

\section{Otra bibliografía}

Asociación Española de Ejecutivos de Finanzas (AEEF) (1999), Primera Cumbre Europa-Mercosur. Madrid.

BANCo InTERAMERICANO DE Desarrollo, 1998. Informe anual. Washington D.C. PROBANCA (1999), La banca española en Iberoamérica. Inversiones y perspectivas. Madrid.

Requeijo, J. (1995), Economía Mundial. Un análisis entre dos siglos. Edit. McGraw Hill, Madrid. 